\title{
PROGRESO DE GÉNERO, ACCESO A LA JUSTICIA Y CAMPAÑAS DE PREVENCIÓN COMO DETERMINANTES DEL FEMINICIDIO EN PERÚ
}

\begin{abstract}
RESUMEN
En los últimos años ha sido notorio el mayor interés del Estado peruano por la problemática de la violencia en contra de la mujer y su escenario extremo, el feminicidio. En ese sentido, empleando un modelo de datos de panel con efectos fijos, para el periodo 2011-2017 y los 24 departamentos del Perú más la Provincia Constitucional del Callao, determinamos el efecto que, sobre la tasa agregada de feminicidios (feminicidios y sus tentativas) tienen las variables indicadas; además de algunas variables macrosociales y del entorno del feminicida. Mostramos que los esfuerzos por mejorar el acceso a la justicia, a través de los centros de emergencia, y el mayor nivel de riqueza departamental, reducen la tasa agregada de feminicidios; mientras que incrementos del número de campañas preventivas y de los ingresos femeninos incrementarían dicha tasa. Variables como el tamaño de la población y la tasa de homicidios dolosos serían también relevantes para explicar incrementos del feminicidio.
\end{abstract}

JEL: J16; D63; B54

PALABRAS CLAVES: Feminicidio; tentativa de feminicidio; progreso de género.

\begin{abstract}
In recent years, the mayor of the Peruvian State has been notorious for the problem of violence against women and its extreme scenario, femicide. In that sense, using a panel data model with fixed effects, for the period 2011-2017 and the 24 departments of Peru plus the Constitutional Province of Callao, determining the effect on the aggregate rate of femicides (femicides and their attempts) have the indicated variables; in addition to some macrosocial variables and the femicide environment. We show that efforts to improve access to justice, through emergency centers, and the higher level of departmental wealth, reduce the aggregate rate of femicides; while increases in the number of preventive campaigns and female income would increase that rate. Variables such as population size and the rate of malicious homicides detected also relevant to explain increases in femicide.
\end{abstract}

JEL: J16; D63; B54

KEYWORDS: Feminicide; attempted Feminicide; Gender Progress

\footnotetext{
A Investigador docente de la Escuela Profesional de Economía de la Facultad de Ciencias Contables, Económicas y Financieras de la Universidad de San Martin de Porres. Funcionario del Organismo Supervisor de Inversión Privada en Telecomunicaciones (Osiptel), Perú. Correo electrónico: jmorim@usmp.pe. El autor agradece el importante aporte de Liz Bautista Cárdenas, estudiante de economía de la USMP, como asistente de la investigación.
} 


\section{INTRODUCCIÓN}

El término feminicidio - derivado del inglés femicide $^{1}$, cuya traducción literal al español sería femicidio, aunque la versión más difundida y popularizada en América Latina sea feminicidio- es un concepto que intenta dar sentido de especificidad al asesinato de mujeres por razones de género, en oposición a conceptos neutrales como homicidio y asesinato de mujeres. En términos de Radford y Russell (1992), el feminicidio es el asesinato misógino de mujeres realizado por hombres; los cuales, según Campbell et al. (2003) estarían motivados principalmente por la decisión de la pareja de terminar la relación, alguna infidelidad (comprobada o no), u otras variables de similar índole.

La especificidad del término halla sustento en la desbalanceada proporción de los homicidios de género en el contexto de pareja, donde se observa que el asesinato de mujeres realizado por hombres es muy superior al asesinato de hombres por parte de mujeres. Por ejemplo, autores como Campbell et al. (2003) sostienen el $50 \%$ de las mujeres y el $6 \%$ de los hombres son asesinados por sus parejas. Este escenario es también documentado por Karbeyaz et al. (2018), quien precisa que, a diferencia de los asesinatos a hombres -donde el homicida es generalmente alguien desconocido-, es más probable que una mujer sea asesinada por alguien que conoce (el asesino suele ser esposo, novio, exesposo o exnovio de la víctima).

En el Perú, la problemática del feminicidio es también preocupante. En este país, representa una de las principales causas de asesinatos dolosos a mujeres [más de $25 \%$ en el año 2017 (INEI, 2019d)]; siendo que, según estadísticas del Ministerio de la Mujer y Poblaciones Vulnerables (MIMP), en promedio durante el periodo 2011 2017 ocurrieron más de 106 feminicidios y más de 171 tentativas de feminicidio al año (en dicho periodo, en total 743 mujeres fueron asesinadas y 1197 sufrieron una tentativa). Esto es, entre los años 2011 y 2017 asesinaron a tres mujeres (o intentaron asesinarlas) por razones de género cada cuatro días.

Según la evidencia descrita, resulta natural que las políticas públicas se orienten a atender este

1 Si bien el término se asocia a Diana Russell (enunciada en el extinto Tribunal Internacional de Crimenes contra la Mujer, llevado a cabo en Bruselas en el año 1976). No obstante, la misma autora le atribuye el término a Carol Orlock (véase Radford y Russell, 1992). problema, por lo que no sorprende que autores como Dobash y Dobash (2004) señalen que estas políticas deben orientarse a cuidar, proteger y prevenir una situación de agresión de una pareja masculina a una víctima femenina, y no un caso contrario.

Los estudios sobre las razones que explican los feminicidios podrían ser analizados desde dos ópticas distintas: i) estudios de casos documentados de feminicidio (enfoque microsocial) y ii) estudios macrosociales de los determinantes del feminicidio (enfoque macrosocial).

Las investigaciones con enfoque microsocial suelen estudiar las características individuales que explican el feminicidio, tanto desde la óptica de la víctima como del victimario. En esa línea, autores como Karbeyaz et al. (2018) analizan las características de casos de feminicidio de los últimos 25 años en Eskisehir, Turquía, donde examinaron los resultados de autopsias, las características sociodemográficas, clínicas, forenses y criminológicas de las víctimas; además de las características de los acusados y sus razones para cometer estos delitos. En similar línea, estudios como los de Campbell et al. (2003), Campbell et al. (2007), Dobash y Dobash (2011) o Johnson et al. (2017) inciden en la relevancia de examinar el feminicidio íntimo desde la perspectiva de los perpetradores, debido a que proporciona información importante sobre los factores individuales, contextuales y situacionales asociados con estos asesinatos.

La literatura descrita encuentra que los factores de riesgo demográfico más importantes en el feminicidio son, como detallan Campbell et al. (2003) -en un estudio de determinación de los factores de riesgo de feminicidio de parejas en relaciones violentas en los Estados Unidos-, el desempleo masculino, el hecho de; que el individuo consuma sustancias tóxicas o sea portador de un arma de fuego o, como adicionalmente advierten Campbell et al. (2007), los antecedentes de violencia en la relación incluidos los intentos de aislar a la víctima y las amenazas previas de matarla- y factores adicionales de violencia como la separación actual o pendiente.

Por otro lado, Dobash y Dobash (2011), en un intento de descubrir una forma efectiva de resocializar a los condenados por feminicidio (estudio sobre 104 archivos de casos de hombres 
condenados por este delito en los años 90 en el Reino Unido), señalan que los hombres, en su mayoría, atribuyen la culpa y la responsabilidad a la mujer víctima por no cumplir con sus estándares de esposa o madre.

Dentro del enfoque macrosocial, la mayoría de los estudios emplean la tasa de feminicidios (en tantos por 100,000), o una transformación de esta, como variable a ser explicada por el modelo, siendo que son recurrentes -aunque no necesariamente analizadas simultáneamente- las variables explicativas asociadas al progreso o desigualdad de género, las políticas de gobierno en torno al acceso a la justicia para mujeres o la concientización del problema de la violencia contra ellas, además de variables macrosociales $y$ otras de índole agregada vinculadas principalmente con las características del victimario.

En ese contexto podemos citar a Palma-Solis et al. (2008), quienes, empleando un modelo de regresión logística multivariante, encuentran un fuerte vínculo del feminicidio con variables macroeconómicas como un menor gasto gubernamental; no obstante, precisan que el riesgo de feminicidio podría ser mayor en contextos en que existe un deficiente progreso de género (desigualdad de género en términos de empleo, educación y representación política). Después de estudiar todos estos indicadores, encuentran que una menor participación femenina en la política surge como el mayor factor de riesgo para el feminicidio. Asimismo, señalan la existencia de evidencia empírica sobre la relación entre el estatus socioeconómico más alto de las mujeres, especialmente en términos de ingresos y empleo, y un aumento en el riesgo de violencia de pareja y feminicidio íntimo. Esta relación contradictoria demuestra, según los autores, que el efecto combinado de los factores relacionados con el progreso económico, político y de género se debe tener en cuenta al tratar el tema de la violencia de pareja contra la mujer y el feminicidio íntimo.

Por su parte, Grana (2001) modela la tasa de feminicidios para 32 estados en los Estados Unidos, considerando tres tipos de variables asociadas principalmente a i) variables de estrés económico y desigualdad, ii) variables de justicia penal y iii) variables comunitarias. El autor encuentra que el tamaño del Estado tiene el mayor impacto en el número de feminicidios domésticos (tal vez debido a la relación entre el tamaño del Estado y otras variables como la pobreza y el acceso a los servicios públicos); asimismo, sugiere que las variables no relacionadas con el feminicidio doméstico son la cantidad de oficiales en la calle, la cantidad de delitos violentos o el ingreso familiar.

Por otro lado, Albuquerque y Vemala (2008) analizan el feminicidio y su relación con la tasa de homicidios masculinos, en el contexto de comparaciones entre ciudades mexicanas fronterizas y no fronterizas con Estados Unidos, además de Los Ángeles y Houston. Mediante el uso de datos de panel para un periodo de seis años (1998-2003), encuentran que las tasas de feminicidio en ciudades aparentemente violentas en términos de homicidios, como Ciudad Juárez, son consistentes con las tasas en ciudades mexicanas menos violentas, en los mismos términos, después de tener en cuenta los efectos de las tasas de homicidios masculinos. Así, encuentran que las ciudades con poblaciones más grandes tienen tasas más altas de homicidios masculinos $\mathrm{y}$, como resultado, también tienen tasas más altas de feminicidios; lo que implica que, dentro de su análisis, la tasa de homicidios masculinos es el factor explicativo más importante de las tasas de feminicidios [tasa de feminicidio como variable explicada frente a la tasa de homicidios masculinos, la población y otras variables de identificación geográfica (como si una ciudad es fronteriza o no)]. Consideran la hipótesis de que las tasas de feminicidio pueden verse afectadas por las tasas de homicidios; no así el caso inverso, con lo que, en el contexto de los autores, la doble causalidad no estaría evidenciada. No obstante, reconocen que sus resultados deben tomarse como "ejercicios estadísticos", debido a que no consideran otros factores socioeconómicos e institucionales que también podrían afectar tanto las tasas de homicidios como de feminicidios masculinos.

En el contexto de Perú, los estudios son principalmente de enfoque macrosocial; siendo pocos $-y$ recientes- los que intentan explicar los efectos de políticas públicas explícitamente orientadas a la disminución de los feminicidios. Así, dentro de estos estudios, podemos citar a:

- Hernández (2015), resalta la problemática de la existencia de dos fuentes de información de feminicidios y tentativas en el Perú, evalúa los determinantes del "feminicidio agregado", concepto que incluye al feminicidio y a las tentativas de feminicidio, justificando su agregación por 
la delgada línea que divide las tentativas de los feminicidios desde un punto de vista epidemiológico y desde la intencionalidad del victimario. El autor considera una función de distribución tipo Poisson de la variable tasa de feminicidio agregado, bajo el argumento de que los feminicidios son un suceso raro; asimismo, aplica la metodología de datos de panel con efectos aleatorios para incorporar diferencias regionales. Encuentra una relación inversa entre el número de mujeres violentadas físicamente y el número de feminicidios agregados; en tanto, concluye que las violencias psicológica y sexual no resultaron buenos predictores del número de feminicidios. También prevé una relación positiva y significativa, aunque débil, entre el número de feminicidios agregados y el ingreso mensual de las mujeres. Lo que sugeriría que la autonomía económica de aquellas podría amenazar la identidad en ciertos hombres. Debe precisarse que como Hernández (2015), Hernández et al. (2018) advierten que el registro de feminicidios en el Perú adolecería de credibilidad (derivado de la divergencia entre los feminicidios y las tentativas de feminicidio reportados por el Observatorio de Criminalidad del Ministerio Público y las estadísticas del Ministerio de la Mujer y Poblaciones Vulnerables -MIMP-).

- Agüero (2019), quien estudia el efecto de las campañas de concientización -de sensibilización o de prevención- sobre las tasas de violencia de pareja hacia las mujeres y el feminicidio en el Perú (evaluación del logaritmo de feminicidios frente a campañas de concientización y el uso de electricidad a nivel departamento). Encuentra que, en el caso donde dos agentes tienen objetivos opuestos, las campañas de información empeoran los resultados. Específicamente, cuestiona la eficacia de la intensidad de las campañas de concientización a nivel nacional para reducir la violencia contra las mujeres en Perú, demostrando que los esfuerzos ahí desplegados llevaron a más violencia, incluyendo más feminicidios. Señala -y documenta- que las campañas de concientización organizadas en Perú están orientadas a definir a los hombres como violentos, lo que podría explicar una sobrerreacción por parte de ellos. Para controlar la posible presencia de causalidad inversa, analiza el uso de días internacionales de observancia como instrumentos plausibles para las campañas (búsquedas en Google de términos "feminicidio" y "violencia en contra de la mujer"), sugiriendo que los efectos de las campañas de sensibilización sobre la violencia en contra de las mujeres son causales.

- Kavanaugh et al. (2019), quienes modelan el acceso a la justicia para mujeres, entendiendo que estas no pueden confiar en el sistema de justicia como un impedimento creíble a la violencia en contra de ellas (sustentado en las altas tasas de subinformación de violencia de género y las bajas tasas de arresto por delitos contra ellas) y, como variable de mejora en el acceso a la justicia, emplean el acceso a los Centros de Emergencia Mujer (CEM). Encuentran que mejorar el acceso a la justicia para las mujeres reduce la violencia doméstica, los feminicidios y las muertes de mujeres debido a la agresión. Esto debido a que los CEM pueden generar una amenaza creíble hacia los perpetradores de una efectiva sanción penal, del mismo modo que permite empoderar a las mujeres como resultado de la confianza de tener acceso a la justicia y los servicios de apoyo más útiles ${ }^{2}$.

Estos estudios referidos a la realidad peruana, se desarrollaron en un contexto de mayor interés del Estado por la problemática del feminicidio, tanto desde el punto de vista legal como institucional. Así, a finales del año 2011 se incorpora en la legislación peruana (Ley $\mathrm{N}^{\circ}$ 29819) la primera referencia sobre feminicidio, mediante la modificación del artículo 107 del Código Penal peruano, agregándose al delito de parricidio el de feminicidio. Sin embargo, al representarse al feminicidio como una variante del primero, no se evidenciaba a este tipo de violencia en el marco de violencia de género descrito en párrafos precedentes; asimismo, limitaba la valoración del tipo penal al de feminicidio íntimo. Esta situación fue superada desde mediados del año 2013 (Ley $\mathrm{N}^{\circ}$ 30068), donde se define al feminicidio como el asesinato de una mujer por su condición de tal (artículo 108-B del Código Penal). Posteriormente, dadas las mejoras en la legislación de este delito, desde mediados del año 2018 (Ley $\mathrm{N}^{\circ}$ 30819) se incorporan agravantes adicionales y se incrementa la pena privativa asociada. ${ }^{3}$

\footnotetext{
2 Las autoras precisan que los CEM mejoran la calidad de la prestación de servicios y su efectividad al permitir que las mujeres sigan todos los pasos de sus quejas en una sola oficina. Además, al existir un mayor número de mujeres policias encargadas de recabar las quejas, aumenta la probabilidad de que otras mujeres se acerquen a los CEM (usualmente (evila presentes en comisaría y otras instituciones gubernamentales) por ayuda.

3 Incorporación del artículo específico sobre feminicidio según
} 
Asimismo, dentro del periodo de análisis, se observan mejoras en las políticas de desarrollo institucional como la creación de centros especializados de atención a mujeres víctimas de violencia [p. ej., los $\mathrm{CEM}^{4}$ dependientes del MIMP $^{5}$, donde solo en el periodo 2011-2017 estos duplicaron su número], la promoción de campañas de concientización sobre la violencia contra la mujer y el feminicidio ( $p$. ej., las Acciones Preventivas Promocionales desarrolladas por los CEM, donde durante el periodo 2011-2017 el número de campañas preventivas se incrementó en $80 \%$ ), políticas de empoderamiento de la mujer en la sociedad peruana como la Ley de cuotas electorales ${ }^{6}$ (a través de la cual la participación femenina en política se incrementó considerablemente, pasando, para el caso de regidoras, de poco más de $8 \%$ a finales de 1990 a $28,5 \%$ en el periodo electoral 2015/2018), entre otras.

Entendiendo la importancia evidenciada por el Estado peruano, y considerando la situación asimétrica de violencia documentada en la literatura, resulta necesario explicar por qué cierto grupo de hombres matan o intentan matar a sus mujeres y, una vez entendido el mayor número de determinantes propuestos, qué es lo que podría hacer el gobierno para mitigar el delito.

Si bien la literatura presentada evalúa variables de política específicas dentro del análisis, son pocos los estudios que evalúan las acciones de gobierno en más de una variable. Esto es, aun cuando se atisba el análisis de ciertas políticas como campañas de prevención, mejoras en el acceso a la justicia para mujeres, o adelantos en el progreso de género, el análisis empírico no suele evaluarlas simultáneamente. En ese sentido, y tomando en consideración la literatura presentada, esta investigación tiene el objetivo de determinar el impacto que en la tasa agregada de feminicidios tienen, variables determinantes

el artículo $2^{\circ}$ de la Ley $\mathrm{N}^{\circ} 30068$ (año 2013), modificado por el Decreto Legislativo $\mathrm{N}^{\circ} 1323$ (año 2017) y modificado por la Ley $\mathrm{N}^{\circ} 30819$ (año 2018).

4 En términos de Kavanaugh et al. (2019), son instituciones estatales especializadas que emplean principalmente a mujeres oficiales en esfuerzos para reducir la violencia de género, al reunir a los servicios policiales, legales y médicos en una sola oficina para integrar todos los pasos del proceso de quejas.

$5 \mathrm{Si}$ bien adopta esta denominación en el año 2012 (Decreto Legislativo $\mathrm{N}^{\circ}$ 1098), este ministerio fue creado en el año 1996 (Decreto Legislativo $\mathrm{N}^{\circ}$ 866) como Ministerio de Promoción de la Mujer y del Desarrollo Humano.

6 Desde el año 1997 (Ley $N^{\circ}$ 26859, Ley de Cuotas Electorales) se establece la cuota de género en el Perú. Esta norma que, inicialmente fijaba una cuota de $25 \%$ de representación de mujeres o varones en las listas parlamentarias y municipales, desde el año 2000 la fija en $30 \%$ (Ley $\mathrm{N}^{\circ} 27387$ ). del progreso de género (como la participación política, el grado de escolaridad y nivel de ingresos femeninos), el acceso a mejor justicia (a través de los CEM) y las campañas realizadas por el MIMP con el objetivo de concientizar a la población sobre la violencia de género, entre otras variables usualmente abordadas por la literatura (variables macrosociales y de entorno del feminicida), además de estimar los efectos inobservables de este tipo de delincuencia.

Además de esta introducción, el artículo consta de tres secciones más: la sección metodológica, la presentación de los principales resultados y, finalmente, la discusión y las conclusiones de rigor.

\section{MÉTODO}

Para concluir si las variables relacionadas con el progreso de género, las mejoras en el acceso a la justicia para las mujeres, las campañas de prevención, entre otras, son determinantes del feminicidio en el Perú, consideramos información longitudinal de mujeres de 15 años a más -si aplica-, de los 24 departamentos del país y la Provincia Constitucional del Callao, durante el periodo 2011-2017.

Para ello, y con el objetivo de superar el poco control de la heterogeneidad observada, presente en la mayoría de los estudios que analizan delitos $^{7}$-p. ej., Grana (2001) documenta problemas de heterogeneidad en la recolección de datos sobre feminicidios a nivel estatal en los Estados Unidos; situación que no mejora incluso cuando los modelos son estimados mediante ecuaciones simultáneas (Cornwell y Trumbull, 1994)-, empleamos datos de panel con efectos fijos individuales (departamentales).

La inclusión de efectos fijos individuales en la regresión de la tasa de feminicidio (o alguna transformación de esta) controla el sesgo por variables omitidas que, si bien resultan relevantes, pueden ser asumidas invariantes en el tiempo. Así, factores culturales vinculados a la tolerancia, a la justificación frente a la violencia en contra la mujer (tolerancia social al maltrato hacia la mujer, aceptación social del trabajo doméstico exclusivo de mujeres, actitudes culturales hacia la agresión a mujeres condicionada al consumo de alcohol o drogas del agresor, etc.), así como variables demográficas (orientación sexual, religión, raza, idioma,

7 Basados en gran medida en datos de corte transversal (Cornwell y Trumbull, 1994). 
grupo de edad, distancia a lugares específicos, entre otros), pueden variar entre departamentos; pero, pueden ser entendidas como constantes en el tiempo dentro de cada uno de ellos.

En ese sentido, la investigación utiliza departamentos como unidad de análisis, en consistencia con el tratamiento del delito presente en Cornwell y Trumbull (1994), y del feminicidio desarrollado en Grana (2001) y Hernández (2015). Y, reconociendo que el evento "tentativa" manifiesta la misma intencionalidad homicida por parte del agresor, pero se determina solo por la pericia de este, consideramos adecuado definir la variable feminicidio agregado como la suma de los feminicidios y las tentativas de feminicidio ocurridas en cada año y departamento específicos. Este tratamiento es consistente con Hernández (2015) y Hernández et al. (2018); no obstante, a diferencia de dichos autores, y con el objetivo de mantener la consistencia en las fuentes, las tentativas de feminicidios y los feminicidios son extraídos desde las estadísticas del MIMP.

Para el desarrollo del modelo se empleó el software STATA 14, presentándose el siguiente modelo a ser validado por la transformación de Box-Cox a la variable dependiente original (, la tasa de feminicidio agregado en tantos por 100 000) frente a las variables explicativas que se describen ${ }^{8}$ :

$$
t f a_{i t}=X_{i t}^{\prime} \beta+Y_{i t}^{\prime} \delta+Z_{i t}^{\prime} \gamma+W_{i t}^{\prime} \theta+\alpha_{i}+\epsilon_{i t}
$$

- $\mathrm{X}_{\mathrm{it}}^{\prime}$ : Variables determinantes del progreso de género (participación política de la mujer como autoridad, mujeres con secundaria completa, ingresos salariales de la mujer), relacionadas con el estudio de Palma-Solis et al. (2008).

- $\mathrm{Y}_{\mathrm{it}}^{\prime}$ : Variables asociadas a las políticas de gobierno con el objetivo de disminuir la tasa de feminicidio, cobertura territorial del CEM y Eventos Preventivos Promocionales realizados por los CEM, vinculadas, respectivamente, a los estudios de Kavanaugh et al. (2019) y Agüero (2019).

- $Z_{\mathrm{it}}^{\prime}$ : Variables macrosociales (nivel de pobreza, tasa de homicidios, población, PBI) y aquellas relativas al feminicida (desempleo masculino, educación de los hombres,

8 La información de las variables, su definición, fuentes y unidades de medida, entre otros datos relevantes, son presentados en el Anexo I. ingresos salariales del hombre), relacionadas con los estudios de Grana (2001), PalmaSolis et al. (2008), Albuquerque y Vemala (2008.)

- $\mathrm{W}_{\mathrm{it}}^{\prime}$ : Variable que refleja los antecedentes de violencia sexual contra la mujer (violencia sexual reciente).

- $\alpha_{i t}^{\prime}$ : Efectos fijos que reflejan características específicas no observables dentro del departamento, que pueden estar correlacionadas con las variables previas $\mathrm{O}$ con el error, pero no varían en el tiempo.

- $\varepsilon_{\mathrm{it}}^{\prime}$ : son términos de perturbación típicos, se supone que están iid con una media cero y una varianza constante $\sigma^{2}$.

Para sustentar la transformación de la variable dependiente, empleamos el método de Box y Cox (1964), que aproxima la forma funcional más apropiada al comportamiento de los datos analizados. Este método consiste en la transformación de la variable dependiente mediante el uso de una familia de transformaciones paramétricas desde $t f a_{i t}$ a $t f a_{i t}{ }^{(\lambda)}$. Donde $\lambda$ define la transformación particular según:

$$
t f a_{i t}{ }^{(\lambda)}=\left\{\begin{array}{c}
\left(t f a_{i t}{ }^{\lambda}-1\right) / \lambda, y \quad \lambda \neq 0 \\
\ln t f a_{i t}, \quad \text { y } \lambda=0
\end{array}\right.
$$

Así, si $\lambda=0$ la transformación recomendada sería el logaritmo de $t f a_{i t}$ mientras que si $\lambda=1$ la transformación sería lineal. No obstante, como Hamilton (2013) señala, encontrar un parámetro de transformación $\lambda$, que sea óptimo en términos de un criterio de probabilidad máxima, no significa necesariamente que las relaciones se hayan linealizado. Este último objetivo podría perseguirse de manera más efectiva utilizando el juicio y la inspección visual, con la posibilidad de diferentes transformaciones para diferentes variables.

Los parámetros del modelo resultante, previa contrastación de la normalidad de la variable dependiente, se estiman mediante Mínimos Cuadrados Generalizados (MCG), empleando errores estándar robustos consistentes con heteroscedasticidad y autocorrelación (CHA).

Para sustentar el modelo de efectos fijos en vez del de efectos aleatorios, se emplea la prueba de Hausman, donde la hipótesis nula es que el modelo preferido es el de efectos aleatorios frente al de efectos fijos. 
Dentro de los modelos candidatos, se eligen aquellos que cumplen con requisitos mínimos como normalidad de los residuos (probados de manera conservadora al $10 \%$ de significancia), obtuvieron mejores indicadores de ajuste (Akaike) y fueron significativos en el mayor número de variables de interés.

\section{RESULTADOS}

En la Tabla $\mathrm{N}^{\circ} 1$ se presentan algunas estadísticas descriptivas que resumen el comportamiento de la tasa de feminicidio $(t f)$ y la tasa de feminicidio agregado $(t f a)$ para cada uno de los departamentos que componen la población analizada durante el periodo 2011-2017.

\section{TABLA $N^{\circ}$ 1: TASAS DE FEMINICIDIO $Y$ FEMINICIDIO AGREGADO 2011-2017}

\begin{tabular}{|c|c|c|}
\hline Departamento & tf $(1 / 100000)$ & tfa $(1 / 100000)$ \\
\hline Amazonas & 0,43 & 2,72 \\
\hline Áncash & 0,71 & 2,03 \\
\hline Apurímac & 0,45 & 1,15 \\
\hline Arequipa & 1,31 & 3,16 \\
\hline Ayacucho & 1,32 & 3,16 \\
\hline Cajamarca & 0,34 & 1,04 \\
\hline Callao & 0,57 & 1,40 \\
\hline Cusco & 0,71 & 2,09 \\
\hline Huancavelica & 0,64 & 2,38 \\
\hline Huánuco & 0,71 & 2,53 \\
\hline Ica & 0,48 & 2,12 \\
\hline Junín & 0,73 & 2,17 \\
\hline La Libertad & 0,52 & 1,32 \\
\hline Lambayeque & 0,45 & 0,56 \\
\hline Lima & 0,78 & 1,81 \\
\hline Loreto & 0,29 & 1,09 \\
\hline Madre de Dios & 2,01 & 5,66 \\
\hline Moquegua & 1,19 & 1,88 \\
\hline Pasco & 1,10 & 3,31 \\
\hline Piura & 0,36 & 0,94 \\
\hline Puno & 0,73 & 1,61 \\
\hline San Martín & 0,37 & 1,42 \\
\hline
\end{tabular}

\begin{tabular}{|c|c|c|}
\hline Tacna & 1,85 & 3,31 \\
\hline Tumbes & 0,65 & 2,47 \\
\hline Ucayali & 0,43 & 1,78 \\
\hline Total & 0,85 & 2,13 \\
\hline
\end{tabular}

Fuente: MIMP

Elaboración propia

En el Perú, para el periodo 2011-2017 la tasa de feminicidio fue de 0,85 , lo que implica que para el periodo evaluado, asesinaron a una mujer por cada 118000 de ellas. Si consideramos las tentativas de feminicidio (tasa de 2,13), más de dos mujeres fueron asesinadas (o intentaron hacerlo) por cada 100000 de ellas.

Dentro de las estadísticas específicas para el periodo 2011-2017, Madre de Dios fue el departamento que presentó la mayor tasa de feminicidio (más de 2 mujeres asesinadas por cada 100000 de ellas) y de feminicidio agregado (más de 5 víctimas por cada 100000 ); seguido de Tacna, donde casi 2 mujeres fueron asesinadas por cada 100000 de ellas y más de 3 fueron víctimas por cada 100000 de ellas.

Caso contrario al reportado para los departamentos de Lambayeque y Piura, donde las tasas de feminicidios agregados fueron de 0,56 y 0,94 , respectivamente. Esto es, en dichos departamentos menos de una mujer fue asesinada (o intentaron asesinarla) por cada 100 000 de ellas.

A continuación, se presenta el modelo finalmente empleado para el cumplimiento del objetivo de investigación: determinar el impacto que, en la tasa agregada de feminicidios, tienen las variables determinantes del progreso de género, acceso a la justicia y Acciones Preventivas llevadas a cabo por el MIMP. Además de las variables objetivo, el modelo controla la tasa agregada de feminicidios mediante la inclusión de variables macrosociales asociadas al departamento y al victimario.

El modelo resultante sigue una transformación en logaritmos de la variable dependiente original, sustentado en la transformación de Box-Cox ${ }^{9}$ a tal variable:

$9 \mathrm{Si}$ bien son eventos raros, como lo reconoce Hernández (2015), la modificación sugerida por la transformación de BoxCox elimina el problema de la no normalidad de la tasa de feminicidio agregado. 
TABLA $\mathbf{N}^{\circ}$ 2: TRANSFORMACIÓN DE BOX COX

\begin{tabular}{|c|c|c|c|c|c|c|}
\hline & & & & $\mathrm{N}^{\circ}$ de obs & $=$ & 167 \\
\hline & & & & LR chi2(10) & $=$ & 84 \\
\hline Log likelihood & $=$ & $-233,04$ & & Prob $>$ chi 2 & $=$ & 0 \\
\hline $\mathrm{tfa}$ & Coef. & Std. Err. & z & $\mathrm{P}>\mathrm{Z}$ & \multicolumn{2}{|c|}{ [95\% Interval Conf.] } \\
\hline /theta & 0,0975 & 0,0761802 & 1,28 & 0,201 & $-0,0519$ & 0,2468 \\
\hline
\end{tabular}

Fuente: Elaboración propia

Por lo tanto, dado que no es posible rechazar la hipótesis nula de que ni a un nivel significancia teórica del $20 \%$, el modelo finalmente empleado es:

$$
\ln \left(t f a_{i t}\right)=X_{i t}^{\prime} \beta+Y_{i t}^{\prime} \delta+Z_{i t}^{\prime} \gamma+W_{i t}^{\prime} \theta+\alpha_{i}+\epsilon_{i t}
$$

La variable dependiente transformada sigue una distribución log-normal (Test de Jarque Bera $\mathrm{JB}=0,53$ y prueba de asimetría y curtosis para normalidad: Prob $>\mathrm{chi}^{2}=0,74$; por lo que es viable la aplicación del método de MCG. En la Tabla $\mathrm{N}^{\circ} 3$ se presentan los resultados del modelo estimado.

\section{TABLA N ${ }^{\circ}$ 3: RESULTADOS DEL MODELO}

\begin{tabular}{|c|c|c|}
\hline v. dependiente: $\ln (t f a)$ & Coef. & p-valor \\
\hline cob_cem & $-0,01187$ & 0,000 \\
\hline acciones & 0,00046 & 0,000 \\
\hline p_alcaldesa & $-0,01699$ & 0,127 \\
\hline sec_com & $-0,01973$ & 0,440 \\
\hline ing_mujer & 0,00103 & 0,089 \\
\hline lnpobreza & 0,66750 & 0,152 \\
\hline lnpob & 10,4712 & 0,000 \\
\hline lnth & 0,25914 & 0,026 \\
\hline$p b i$ & $-0,00001$ & 0,000 \\
\hline desempleo_hombre & 0,06736 & 0,276 \\
\hline$C$ & $-71,9322$ & 0,000 \\
\hline $\bar{R}^{2}$ & 0,322 & \\
\hline$A I K$ & 241,50 & \\
\hline$F$ & 43,33 & \\
\hline$n$ & 175 & \\
\hline Hausman $\left(\right.$ Prob $\left.>c h i^{2}\right)$ & 0,010 & \\
\hline$J B$ & 3,322 & \\
\hline sktest $\left(\right.$ Prob $\left.>c h i^{2}\right)$ & 0,171 & \\
\hline
\end{tabular}

Fuente: Elaboración propia

A nivel global, las variables incluidas en el modelo explican el $32,2 \%$ de las variaciones en la variable $\ln (\boldsymbol{t} \boldsymbol{f a})$; en tanto, las variables son en conjunto relevantes para explicar el modelo $(F=43,3)$. Asimismo, desde la prueba de Hausman, no ha sido posible concluir sobre una preferencia hacia modelos de efectos aleatorios, por lo que se recomienda el uso del modelo de datos de panel con efectos fijos. Por otro lado, los residuos correspondientes resultan normales. La situación descrita es consistente con la mayoría de los modelos presentados en el Anexo II.

A nivel de cada variable y para el periodo 20112017, los resultados son:

- cob_cem: significativa al 99\%. Así, por cada incremento en la cobertura de los CEM de 10 $000 \mathrm{Km}^{2}$, se espera que la $\boldsymbol{t} \boldsymbol{f a}$ disminuya en $1,19 \%$.

- acciones: significativa al 99\%. Implica que, por cada acción preventiva promocional adicional que se realice, la $\boldsymbol{t} \boldsymbol{f a}$ se incrementaría en $0,046 \%$.

- p_alcaldesa y sec_com: no significativas al $10 \%$ de significancia.

- ing_mujer: significativa al 90\%. Implica que, ante el incremento de un sol en el ingreso salarial femenino, la tfa lo hará en $0,103 \%$.

- Inpobreza: no significativa al $10 \%$ de significancia.

- Inpob: significativa al 99\%. Así, ante incrementos de $1 \%$ en la población, se espera que la $\boldsymbol{t} \boldsymbol{f a}$ se incremente más de $10 \%$.

- Inth: significativa al 95\%, lo que implica que ante un incremento de $1 \%$ la tasa de homicidios dolosos, se espera un incremento de $0,26 \%$ en la $t \boldsymbol{f a}$.

- pbi: significativa al 99\%. Implica que, ante incrementos unitarios del PBI (millones de S/ corrientes), se esperaría una disminución de $0,001 \%$ en la $t f a$.

- desempleo_hombre: variable no significativa al $10 \%$ de significancia.

Adicionalmente a las variables listadas, en modelos alternativos se incorporaron variables relativas al nivel educativo e ingresos de los hombres, además de antecedentes de violencia sexual; sin embargo, los resultados no fueron significativos y empeoraron los resultados del modelo. 


\section{DISCUSIÓN Y CONCLUSIONES}

La discusión de los resultados y las principales conclusiones relacionadas al impacto que, en la tasa agregada de feminicidios tienen, las variables: acceso a mejor justicia, campañas de prevención y progreso de género, son:

- cob_cem: el coeficiente presenta el signo esperado, implica que la mayor cobertura de los CEM (por cada $10000 \mathrm{Km}^{2}$ ) podría tener un efecto disuasivo en los feminicidas. Así, se configurarían como amenazas creíbles, debido a que en estos CEM, adicionalmente a propiciar que las mujeres presenten denuncias por violencia física, psicológica o sexual, estas pueden recibir asesorías y seguimiento de sus casos a nivel judicial. Si bien los resultados obtenidos son consistentes con lo encontrado por Kavanaugh et al. (2019); debe precisarse que las autoras encuentran un coeficiente un tanto mayor de -0,075 (significativa al 5\%) para el periodo 2007-2014 en un modelo log-lineal, debido a que, adicionalmente a la variable indicada, controlan el logaritmo de la tasa de feminicidio -no agregado- por el tamaño de la población. Debe precisarse que las autoras no presentan indicadores de bondad de ajuste de su modelo, tampoco sustentan el uso del modelo de efectos fijos. Finalmente, en este extremo, se recomienda que las acciones de gobierno puedan encaminarse a ampliar la cobertura de los CEM mediante la creación de un número mayor de estos, sobre todo en localidades donde su presencia es limitada.

- acciones: Implica que por cada acción preventiva adicional que se realice, la $t \boldsymbol{f a}$ se incrementaría en $0,046 \%$. Esto es, las acciones de difusión estarían propiciando mayores feminicidios agregados. Una explicación a este resultado sería que las campañas de concientización organizadas en Perú, al estar orientadas a definir a los hombres como violentos, podría generar una sobrerreacción opuesta por parte de ellos. Este resultado, aparentemente contradictorio con la lógica económica, resulta consistente con la literatura donde, por ejemplo, Agüero (2019) encuentra una elasticidad de 0,46 de las campañas sobre el número de feminicidios reportados por el INEI (significativa al 1\%). Debe precisarse que los autores presentan un $\overline{\mathrm{R}}^{2}$ de $10.7 \%$; no obstante, no justifican el uso del modelo de efectos fijos que emplean. Como recomendación de política, se sugiere una revisión del enfoque de las campañas preventivas desde el punto de vista psicológico, a fin de determinar los mecanismos más propicios para la no afectación de las mujeres.

- p_alcaldesa y sec_com : Si bien no resultan significativas (significativa a nivel teórico del $12.7 \%$ y $44 \%$, respectivamente), presentan los signos esperados.

- ing_mujer: el resultado obtenido es aparentemente contrario con el valor esperado; pero consistente con autores como Palma-Solís et al. (2008), o Kavanaugh et al. (2019), quienes precisan que, si bien se suele considerar que la mejora en el estatus económico de las mujeres aliviará la violencia de género, no necesariamente es así.

Asimismo, respecto a otras variables usualmente abordadas por la literatura (variables macrosociales y de entorno del feminicida), es posible concluir:

- Inpobreza y desempleo_hombre: si bien no resultaron significativas, el coeficiente obtenido para cada una de ellas presenta el signo esperado.

- Inpob y phi: ambas variables significativas y con el signo esperado. Así, mientras más grande sea un departamento, mayor sería la $\boldsymbol{t} \boldsymbol{f} \boldsymbol{a}$; en tanto, existe evidencia para concluir que mayores niveles de producto de un departamento implican menores $\boldsymbol{t} \boldsymbol{f a}$.

- Inth: el resultado obtenido sugiere que las tasas de homicidios dolosos son relevantes para explicar la tfa en Perú, lo que sugiere que una disminución de los homicidios dolosos podría reportar una disminución relevante de los feminicidios. Este resultado es consistente con Albuquerque y Vemala (2008), quienes encuentran que incrementos unitarios en la tasa de homicidios implican incrementos de 0,11 puntos (en tanto por 100 000) en la tasa de feminicidios. Los autores reportan un de $\overline{\mathrm{R}}^{2} 55 \%$; no obstante, no justifican el uso del modelo de efectos fijos que emplean. Esto último podría describir al feminicidio como parte de un contexto general de mayor criminalidad y violencia en el país $y$, no necesariamente, como un problema de odio irrestricto hacia las mujeres.

Por otro lado, se advierten algunas limitaciones del estudio:

- No se ha testeado la posible "endogeneidad" derivada de las relaciones entre la "tasa de feminicidio agregado" y las variables "cob_ 
cem" y "acciones". No obstante, siguiendo a Hernández et al. (2018) ${ }^{10}$, Agüero (2019) ${ }^{11}$ y Kavanaugh et al. $(2019)^{12}$, no necesariamente se configuraría como problema.

- No se han considerado algunas variables que posiblemente cambien con el tiempo y que estarían correlacionadas con la variable explicativa. Como aquellas que medirían el efecto disuasorio (penas, capturas y condenas efectivas) en el feminicida o potencial feminicida -distintas a aquellas controladas por los CEM- [P. ej. Campbell et al. (2007) encontraron que los antecedentes de arrestos del agresor aumentan el riesgo de feminicidio], las cuales se asumen aquí, invariantes en el tiempo ${ }^{13}$.

- No se han considerado variables como el número de policías, debido a que según datos del INEI (2019e) la mayoría de los feminicidios y tentativas se dan en el hogar $(57 \%)$. Este argumento resulta consistente con Grana (2001) quien encuentra que la variable número de policías per cápita no resulta significativa.

- No se han incluido variables que midan el "registro de antecedentes de violencia familiar", debido a que podrían adolecer de sesgo de no reporte o el sesgo de reporte no necesariamente vinculado a los objetivos de este estudio.

\section{REFERENCIAS}

[1] Agüero, J. M. (2019). Information and Behavioral Responses with More than One Agent: The Case of Domestic Violence Awareness Campaigns. Connecticut. University of Connecticut, working paper 2019-04.

[2] Albuquerque, P. H., y Vemala, P. R. (2008). A Statistical Evaluation of Femicide Rates in Mexican Cities along the US-Mexico Border. Inédito.

10 Los autores señalan que la relación de doble sentido entre la instalación de los CEM y la mayor violencia no tendría mayor sustento; debido a que el objetivo de mayor cobertura del MIMP.

11 Presenta evidencia de la existencia de un aumento significativo (y el más importante) en las campañas de prevención producida por variables no relacionadas con el feminicidio en el Perú, como las búsquedas en Google de términos relacionados en fechas fijadas internacionalmente.

12 Las autoras muestran que la colocación de un CEM no habria sido anticipada por los cambios en la violencia.

13 Consideramos que las variables determinantes de la disuasión de estas conductas tienen un alcance nacional, por lo que este efecto puede ser internalizado en el modelo de efectos fijos individuales.
[3] Box, G., y Cox, D. (1964). An analysis of transformations. Journal of the Royal Statistical Society. Series B (Methodological), 26(2), 211-252.

[4] Campbell, J. C., Glass, N., Sharps, P. W., Laughon, K., y Bloom, T. (2007). Intimate partner homicide: Review and implications of research and policy. Trauma, Violence \& Abuse, 8(3), 246-269.

[5] Campbell, J. C., Webster, D., KoziolMcLain, J., Block,C., Campbell, D., Curry, M.,..., Laughon, K. (2003). Risk Factors for Femicide in Abusive Relationships: Results from a Multisite Case Control Study. American Journal of Public Health, 93(7), 1089-1097.

[6] Cornwell, C., y Trumbull, W. (1994). Estimating the Economic Model of Crime with Panel Data. The Review of Economics and Statistics, 76(2), 360-366.

[7] Dobash, R. E., y Dobash, R. P. (2011). What Were They Thinking? Men Who Murder an Intimate Partner. Violence Against Women, 17(1), 111-134.

[8] Dobash, R. P., y Dobash, R. E. (2004). Women's violence to men in intimate relationships: Working on a puzzle. British Journal of Criminology, 44(3), 324-349.

[9] Grana, S. J. (2001). Sociostructural Considerations of Domestic Femicide. Journal of Family Violence, 16(4), 421435.

[10] Hamilton, L. (2013). Statistics with Stata (version 12). Boston: Cengage Learning.

[11] Hernández, W. (2015). Feminicidio agregado en el Perú y su relación con variables macrosociales. Revista Latinoamericana de Estudios de Seguridad, (17), 48-66.

[12] Hernández W., Raguz, M., Morales, H., y Burga, A. (2018). Feminicidios determinantes y evaluación de riesgo [informe final]. Universidad de Lima, Consorcio de Investigación Económica y Social.

[13] Instituto Nacional de Estadística e Informática del Perú. (INEI, 2019a). Estadísticas de empleo. Recuperado el 15 de julio de 2019, de https://www.inei.gob. pe/estadisticas/indice-tematico/ocupacion-yvivienda/ 
[14] Instituto Nacional de Estadística e Informática del Perú. (INEI, 2019b). Estadísticas de población. Recuperado el 15 de julio de 2019, de http://proyectos.inei. gob.pe/web/poblacion/

[15] Instituto Nacional de Estadística e Informática del Perú. (INEI, 2019c). Estadísticas de pobreza. Recuperado el 15 de julio de 2019, de https://www.inei.gob.pe/ estadisticas/indice-tematico/poverty/

[16] Instituto Nacional de Estadística e Informática del Perú. (INEI, 2019d). Homicidios en el Perú, contándolos uno a uno 2011-2017, Informe estadístico $N^{\circ} 6$. Lima: autor.

[17] Instituto Nacional de Estadística e Informática del Perú. (INEI, 2019e). Perú: Brechas de Género 2018. Avances hacia la igualdad de mujeres y hombres. Lima: autor.

[18] Johnson, H., Eriksson, L., Mazerolle, P., y Wortley, R. (2017). Intimate Femicide: The Role of Coercive Control. Feminist Criminology, 14(1), 1-21.

[19] Karbeyaz, K., Yetiş, Y., Güneş, A., y Şimşek, Ü. (2018). Intimate Partner Femicide in Eskisehir, Turkey 25 Years Analysis. Journal of Forensic and Legal Medicine, 60, 56-60.
[20] Kavanaugh, G., Sviatschi, M., y Trako, I. (2019). Female Officers, Gender Violence and Human Capital: Evidence from All-Women's Justice Centers in Peru. Disponible en: https://ssrn.com/ abstract $=3022670$

[21] Ministerio de la Mujer y Poblaciones Vulnerables. (MIMP, 2019a). Programa Nacional Contra la Violencia Familiar y Sexual. Boletines y Resúmenes estadísticos 2011-2017. Recuperado el 29 de junio de 2019, de https:/www.mimp.gob.pe/contigo/ contenidos/pncontigo-articulos.php? codigo=32

[22] Ministerio de la Mujer y Poblaciones Vulnerables (MIMP, 2019b). Programa Nacional Contra la Violencia Familiar y Sexual. Estadísticas - Prevención de la Violencia 2011-2017. Recuperado el 29 de junio de 2019, de https://www.mimp.gob. pe/contigo/contenidos/pncontigo-articulos. php? codigo $=33$

[23] Palma-Solis, M., Vives-Cases, C., y Álvarez-Darnet, C. (2008). Gender Progress and Government Expenditure as Determinants of Femicide. Ann Epidemiol, 18(4), 322-329.

[24] Radford, J., y Russell, D. (1992). Femicide, the politics of woman killing, Nueva York: Twayne Publishers. 


\section{ANEXO I. VARIABLES EMPLEADAS}

\begin{tabular}{|c|c|c|c|}
\hline Variable & Definición & Fuente & $\begin{array}{l}\text { Unidad de } \\
\text { medida }\end{array}$ \\
\hline Tasa de feminicidio (tf) & $\begin{array}{l}\text { Cociente del número absoluto de feminicidios multiplicado } \\
\text { por } 100000 \text { sobre la población femenina anual del } \\
\text { departamento evaluado. No se toma en cuenta la distinción } \\
\text { entre un feminicidio íntimo y el feminicidio no íntimo. }\end{array}$ & $\begin{array}{l}\text { MIMP (2019). Programa Nacional Contra la Violencia Familiar y Sexual. Dis- } \\
\text { ponible en: https://www.mimp.gob.pe/contigo/contenidos/pncontigo-articulos. } \\
\text { php?codigo=33 }\end{array}$ & $\begin{array}{c}\text { Tasa } \\
\text { x100 } 000\end{array}$ \\
\hline Tasa agregada de feminicidio (tfa) & $\begin{array}{l}\text { Cociente de la suma del número absoluto de feminicidios y } \\
\text { del número absoluto de tentativas de feminicidio multipli- } \\
\text { cados por } 100000 \text { sobre la población femenina anual del } \\
\text { departamento evaluado. No se toma en cuenta la distinción } \\
\text { entre un feminicidio íntimo y el feminicidio no íntimo. }\end{array}$ & $\begin{array}{l}\text { MIMP (2019). Programa Nacional Contra la Violencia Familiar y Sexual. Dis- } \\
\text { ponible en: https://www.mimp.gob.pe/contigo/contenidos/pncontigo-articulos. } \\
\text { php?codigo=33 }\end{array}$ & $\begin{array}{c}\text { Tasa } \\
\mathrm{x} 100000\end{array}$ \\
\hline $\begin{array}{l}\text { Cobertura territorial del CEM } \\
\text { (cob_cem) }\end{array}$ & $\begin{array}{l}\text { Cociente del número de Centros de Emergencia Mujer sobre } \\
\text { la extensión del departamento. }\end{array}$ & $\begin{array}{l}\text { MIMP (2019). Programa Nacional Contra la Violencia Familiar y Sexual. Dis- } \\
\text { ponible en: https://www.mimp.gob.pe/contigo/contenidos/pncontigo-articulos. } \\
\text { php?codigo=33 } \\
\text { INEI: extensión territorial. }\end{array}$ & $\begin{array}{l}\text { CEM por cada } \\
10000 \mathrm{Km} 2\end{array}$ \\
\hline $\begin{array}{l}\text { Eventos Preventivos Promocionales } \\
\text { realizados por los CEM (acciones) }\end{array}$ & $\begin{array}{l}\text { Número de Acciones Preventivas Promocionales realizados } \\
\text { por los CEM (campañas de concientización) }\end{array}$ & $\begin{array}{l}\text { MIMP (2019). Programa Nacional Contra la Violencia Familiar y Sexual. Dis- } \\
\text { ponible en: https://www.mimp.gob.pe/contigo/contenidos/pncontigo-articulos. } \\
\text { php?codigo=32 }\end{array}$ & Número natural \\
\hline $\begin{array}{l}\text { Participación política de la mujer } \\
\text { como autoridad (p_alcaldesa) }\end{array}$ & $\begin{array}{l}\text { Porcentaje de mujeres alcaldesas respecto al total de alcaldes } \\
\text { y alcaldesas }\end{array}$ & $\begin{array}{l}\text { INEI (2019). Perú: Brechas de Género 2018. Avances hacia la igualdad de } \\
\text { mujeres y hombres. }\end{array}$ & Porcentaje (\%) \\
\hline $\begin{array}{l}\text { Mujeres con secundaria completa } \\
\text { (sec_com) }\end{array}$ & $\begin{array}{l}\text { Mujeres de } 17 \text { a más años de edad con al menos estudios } \\
\text { secundarios completos }\end{array}$ & $\begin{array}{l}\text { INEI (2019). Perú: Brechas de Género 2018. Avances hacia la igualdad de } \\
\text { mujeres y hombres. }\end{array}$ & $\%$ \\
\hline $\begin{array}{l}\text { Ingresos salariales de la mujer } \\
\text { (ing_mujer) }\end{array}$ & $\begin{array}{l}\text { Ingreso promedio mensual proveniente del trabajo de mu- } \\
\text { jeres }\end{array}$ & $\begin{array}{l}\text { INEI (2019). Perú: Brechas de Género 2018. Avances hacia la igualdad de } \\
\text { mujeres y hombres. }\end{array}$ & $\begin{array}{l}\text { Soles }(\mathrm{S} / \text { ) co- } \\
\text { rrientes }\end{array}$ \\
\hline Nivel de pobreza (pobreza) & $\begin{array}{l}\text { Porcentaje de la población con al menos una necesidad bási- } \\
\text { ca insatisfecha }\end{array}$ & $\begin{array}{l}\text { INEI (2019). Estadísticas de pobreza. Disponible en: https://www.inei.gob.pe/ } \\
\text { estadisticas/indice-tematico/poverty/ }\end{array}$ & $\%$ \\
\hline $\begin{array}{l}\text { Desempleo masculino (desempleo } \\
\text { hombre) }\end{array}$ & PEA ocupada hombre/PEA total hombre & $\begin{array}{l}\text { INEI (2019). Perú: Brechas de Género 2018. Avances hacia la igualdad de } \\
\text { mujeres y hombres. }\end{array}$ & $\%$ \\
\hline
\end{tabular}




\begin{tabular}{|c|c|c|c|}
\hline Variable & Definición & Fuente & $\begin{array}{l}\text { Unidad de } \\
\text { medida }\end{array}$ \\
\hline Tasa de homicidios (th)* & $\begin{array}{l}\text { Muertes violentas asociadas a eventos delictivos dolosos por } \\
100000 \text { sobre la población del departamento }\end{array}$ & $\begin{array}{l}\text { INEI (2019). Homicidios en el Perú, contándolos uno a uno 2011-2017. Infor- } \\
\text { me estadístico N }{ }^{\circ} 6 \text {. Lima: autor. }\end{array}$ & $\begin{array}{c}\text { Tasa } \\
\times 100000\end{array}$ \\
\hline Población (pob) & Habitantes por departamento & $\begin{array}{l}\text { INEI (2019). Proyecciones de población. Disponible en: http://proyectos.inei. } \\
\text { gob.pe/web/poblacion/ }\end{array}$ & $\begin{array}{l}\text { Miles de habi- } \\
\quad \text { tantes }\end{array}$ \\
\hline Producto Bruto Interno (pbi) * & PBI nominal & $\begin{array}{l}\text { INEI (2019). Producto Bruto Interno por departamentos 2011-2017. Disponible } \\
\text { en: https://www.inei.gob.pe/estadisticas/indice-tematico/economia/ }\end{array}$ & $\begin{array}{l}\text { Millones de } \mathrm{S} / \\
\text { corrientes }\end{array}$ \\
\hline $\begin{array}{l}\text { Educación de los hombres (educ_ } \\
\text { hombre) }\end{array}$ & Años de educación de los hombres & $\begin{array}{l}\text { INEI (2019). Perú: Brechas de Género 2018. Avances hacia la igualdad de } \\
\text { mujeres y hombres. }\end{array}$ & Número natural \\
\hline $\begin{array}{l}\text { Ingresos salariales del hombre } \\
\text { (ing_hombre) }\end{array}$ & $\begin{array}{l}\text { Ingreso promedio mensual proveniente del trabajo de hom- } \\
\text { bres }\end{array}$ & $\begin{array}{l}\text { INEI (2019). Perú: Brechas de Género 2018. Avances hacia la igualdad de } \\
\text { mujeres y hombres. }\end{array}$ & $\begin{array}{l}\text { Soles (S/) co- } \\
\text { rrientes }\end{array}$ \\
\hline $\begin{array}{l}\text { Violencia sexual reciente (viol_ } \\
\text { sex_rec) }\end{array}$ & $\begin{array}{l}\text { Violencia sexual ejercida por el esposo o compañero en los } \\
\text { últimos } 12 \text { meses }\end{array}$ & $\begin{array}{l}\text { INEI (2019). Perú: Brechas de Género 2018. Avances hacia la igualdad de } \\
\text { mujeres y hombres. }\end{array}$ & $\%$ \\
\hline
\end{tabular}

* Las cifras para los años 2016 y 2017, según lo indica el INEI, son estimadas. 
ANEXO II. REGRESIONES DEL FRENTE A DISTINTAS VARIABLES EXPLICATIVAS.

\begin{tabular}{|c|c|c|c|c|c|c|c|c|c|}
\hline Variable & E1 & E2 & E3 & E4 & E5 & E6 & E7 & E8 & E9 \\
\hline cob_cem &,$- 003024 *$ &,$- 013951 * * *$ &,$- 013629 * * *$ &,$- 011399 * * *$ &,$- 011223^{* * *}$ &,$- 014679 * * *$ &,$- 014409 * * *$ &,$- 011922 * * *$ &,$- 012189 * * *$ \\
\hline acciones &, $000462 * *$ &, $000459^{* * *}$ &, $000456 * * *$ &, $000474 * * *$ &, $00047 * * *$ &, $000453 * * *$ &, $000448 * * *$ &, $000463 * * *$ &, $000449 * * *$ \\
\hline p_alcaldesa &,$- 03015^{*}$ & & & $-0,016709$ & $-0,015866$ & & & $-0,016878$ & $-0,017836$ \\
\hline sec_com & 0,001257 & & $-0,018181$ & & $-0,017193$ & & $-0,02066$ & $-0,019818$ & $-0,023404$ \\
\hline ing_mujer & ,000991* & 0,00056 & 0,000822 & 0,000699 & ,000939* & 0,000594 & 0,000897 &, $001031^{*}$ & 0,000831 \\
\hline lnpobreza & &, $811382 *$ & 0,721185 &, $824461^{*}$ & 0,738506 & 0,76165 & 0,652596 & 0,665059 & 0,721224 \\
\hline lnpob & & $11,2178^{* * *}$ & $11,3292 * * *$ & $10,3024 * * *$ & $10,454 * * *$ & $11,2711 * * *$ & $11,4047 * * *$ & $10,6487 * * *$ & $10,5089 * * *$ \\
\hline lnth & &, $235086^{* *}$ &, $247293^{* *}$ &, $235411^{* *}$ &, $246938^{* *}$ &, $243872 * *$ &, $258903 * *$ &, $255337 * *$ &, $255925 * *$ \\
\hline $\mathrm{pbi}$ & & $-8,7 \mathrm{e}-06^{* * *}$ & $-9,3 \mathrm{e}-06^{* * *}$ & $-9,4 \mathrm{e}-06^{* * *}$ & $-9,9 \mathrm{e}-06^{* * *}$ & $-9,7 \mathrm{e}-06^{* * *}$ &,$- 000011 * * *$ &,$- 000011^{* * *}$ &,$- 000012 * * *$ \\
\hline desempleo_hombre & & & & & & 0,056507 & 0,063961 & 0,069098 & 0,068408 \\
\hline educ_hombre & & & & & & & & & 0,085069 \\
\hline ing_hombre & & & & & & & & & 0,000315 \\
\hline viol_sex_rec & & & & & & & & 0,01161 & \\
\hline $\mathrm{C}$ & $-0,719252$ & $-77,7661^{* * *}$ & $-77,5669 * * *$ & $-71,8018^{* * *}$ & $-71,9143 * * *$ & $-78,1422^{* * *}$ & $-77,9655^{* * *}$ & $-73,142 * * *$ & $-73,2104 * * *$ \\
\hline \multirow[t]{2}{*}{ r2_a } & 0,205 & 0,322 & 0,321 & 0,322 & 0,321 & 0,321 & 0,322 & 0,318 & 0,315 \\
\hline & $\begin{array}{c}\text { aic } \\
348,64 \\
238,77 \\
239,82\end{array}$ & & & 239,75 & 240,89 & 239,78 & 240,56 & 243,40 & 245,11 \\
\hline $\mathrm{F}$ & 30,27 & 63,02 & 59,94 & 58,58 & 56,13 & 47,70 & 44,96 & 37,57 & 43,73 \\
\hline $\mathrm{N}$ & 225 & 175 & 175 & 175 & 175 & 175 & 175 & 175 & 175 \\
\hline Hausman (Prob>chi2) & 0,019 & 0,000 & 0,000 & 0,003 & 0,005 & 0,001 & 0,001 & 0,010 & 0,033 \\
\hline $\mathrm{JB}$ & 19,291 & 5,464 & 4,422 & 4,638 & 3,722 & 5,234 & 4,077 & 3,390 & 3,322 \\
\hline sktest (Prob>chi2) & 0,001 & 0,075 & 0,109 & 0,100 & 0,146 & 0,080 & 0,125 & 0,166 & 0,171 \\
\hline
\end{tabular}

